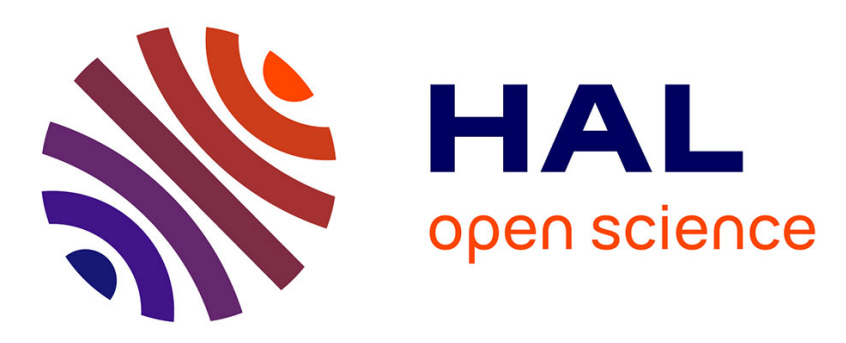

\title{
Structure refinement of decagonal Al-Ni-Co, superstructure type I
}

Pawel Kuczera, Janusz Wolny, Frank Fleischer, Walter Steurer

\section{To cite this version:}

Pawel Kuczera, Janusz Wolny, Frank Fleischer, Walter Steurer. Structure refinement of decagonal AlNi-Co, superstructure type I. Philosophical Magazine, 2010, pp.1. 10.1080/14786435.2010.518985 . hal-00631267

\section{HAL Id: hal-00631267 https://hal.science/hal-00631267}

Submitted on 12 Oct 2011

HAL is a multi-disciplinary open access archive for the deposit and dissemination of scientific research documents, whether they are published or not. The documents may come from teaching and research institutions in France or abroad, or from public or private research centers.
L'archive ouverte pluridisciplinaire HAL, est destinée au dépôt et à la diffusion de documents scientifiques de niveau recherche, publiés ou non, émanant des établissements d'enseignement et de recherche français ou étrangers, des laboratoires publics ou privés. 


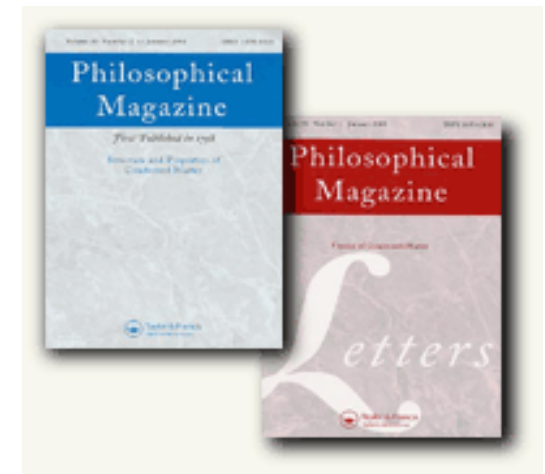

\section{Structure refinement of decagonal Al-Ni-Co, superstructure type I}

\begin{tabular}{|c|c|}
\hline Journal: & Philosophical Magazine \& Philosophical Magazine Letters \\
\hline Manuscript ID: & TPHM-10-Jun-0262.R1 \\
\hline Journal Selection: & Philosophical Magazine \\
\hline $\begin{array}{r}\text { Date Submitted by the } \\
\text { Author: }\end{array}$ & 02-Aug-2010 \\
\hline Complete List of Authors: & $\begin{array}{l}\text { Kuczera, Pawel; AGH University of Science and Technology, of } \\
\text { Physics and Applied Computer Science } \\
\text { Wolny, Janusz; AGH University of Science and Technology, Faculty } \\
\text { of Physics and Applied Computer Science } \\
\text { Fleischer, Frank; Laboratory of Crystallography, ETH Zurich } \\
\text { Steurer, Walter; ETH Zurich, Department of Materials }\end{array}$ \\
\hline Keywords: & quasicrystals, structure analysis \\
\hline Keywords (user supplied): & Edagawa phase, Superstructure type I, decagonal quasicrystals \\
\hline
\end{tabular}

\section{SCHOLARONE Manuscripts}




\section{Introduction}

During the last two decades the phase diagram of Al-Ni-Co has been thoroughly investigated. There are eight different modifications of the decagonal phase reported in this system [ 1 and references therein]. One of them is the so called superstructure type I [2]). The diffraction pattern of the superstructure type I phase consists of strong main reflections and rather weak satellite reflections. The satellite reflections cannot be indexed in the standard decagonal reciprocal lattice basis, i.e. the one used for indexing the so called basic Ni-rich phase. Instead, one has to use a rotoscaled basis [3] (rotated by an angle of $\pi / 10$ and rescaled by the factor of $\sqrt{3-\tau}$.Insuch a basis all reflections can be divided into three classes according to: $\left(\sum_{i=1}^{4} h_{i}\right)$ modulo $5=m$ $m= \pm 1$ first order satellites (S1) and with $m= \pm 2$ second order satellites (S2).No quantitative description of this phase has been published yet.

We present the first refinement results of the Edagawa phase conducted in the physical space only. The structure factor used for the modelling process was calculated on the basis of a statistical approach described in [4,5], which allows a purely 3-dimensional, real space optimization of a quasicrystalline structure. This approach has already been successful in the structure refinement of the basic Ni-rich phase[6,7].

\section{Diffraction data set and sample details}

An alloy with nominal composition $\mathrm{Al}_{72.0} \mathrm{Co}_{12.4} \mathrm{Ni}_{15.6}$ was prepared by arc melting of the pure elements and heat treated afterwards. According to the phase diagram, the sample was quenched at $730^{\circ} \mathrm{C}$. The chemical composition was determined to $\mathrm{Al}_{70.6(3)} \mathrm{Co}_{13.3(3)} \mathrm{Ni}_{16.1(2)}$ using a JEOL JXA-8200 WD/ED combined microprobe. Backscattered electron images identified the alloy as single phase. 
Single crystal X-ray diffraction experiments were performed at the SwissNorwegian beam line (SNBL) at the European Synchrotron Radiation Facility (ESRF) in Grenoble, France, using a mar345 image plate detector system (wavelength $0.69393 \AA$, oscillation angle $0.7^{\circ}$ ). Two data sets were collected, one data set (280 frames) with a shortexposure time to prevent saturation of strong reflections and one data set with a longexposure time (244 frames) in order to collect sufficient data for rather weak superstructure reflections. The strongest reflections in the synchrotron diffraction data were still in saturation. Therefore, an in-house data set (974 frames, oscillation angle $0.5^{\circ}$ ) was collected with a four-circle diffractometer equipped with a charge-couple detector system (Xcalibur Oxford Diffraction, $\mathrm{MoK}_{\alpha}$ radiation, graphite monochromator).

All three data sets were processed and integrated with the CrysAlisRED software (Oxford Diffraction) and scaled afterwards. The number of reflections present in all three datasets was sufficiently large for obtaining a reliable scale factor.A total number of over 97,000 reflections was merged in the Laue group $10 / \mathrm{m}$ with $R_{\text {int }}=0.084$. As a result, we obtained a set of 6843 unique reflections(1387 main reflections and 5456 satellite reflections). There were 702 reflections with zero intensity, which were also used in the refinement process. The optimization was performed against structure factor amplitudes, the standard deviations for strong

reflections were calculated as $\sigma\left|F_{o}\right|=\frac{\sigma\left(F_{0}^{2}\right)}{2\left|F_{o}\right|}$ and for weak ones as $\sigma\left|F_{o}\right|=\frac{\sqrt{\sigma\left(F_{0}^{2}\right)}}{2}$ [8]. For 2284 reflections $F$ was greater than $3 \sigma_{F}$.

\section{The structure model}

The initial model was built based on the electron density maps obtained from the charge flipping procedure. These maps correspond well to the model proposed by Yamamoto and Weber [9]. This model consists of decagonal clusters decorating the vertices of a rhombic Penrose tiling. The edge length of rhombuses is around $19 \AA$ andthe proposed space group is centrosymmetric $\mathrm{P} 10_{5} / \mathrm{mcm}$.

Based on two independent structure units, i.e. a thick and a thin rhombus, reasonable results can be obtained only for a refinement against the main reflections. However for main reflections only the $\boldsymbol{h}_{\mathbf{i}} \boldsymbol{h}_{\mathbf{2}} \boldsymbol{h}_{\mathbf{2}} \boldsymbol{h}_{\mathbf{i}} \boldsymbol{h}_{\mathbf{5}}: \boldsymbol{h}_{\mathbf{3}}=\mathbf{2} n+\mathbf{1}$ systematic absences rule was verified.Refinements based on models with non-centrosymmetric space group and four independent structure units, i.e. two pairs of thick and thin rhombuses, gave much better results. A modification of Penrose tiling, that is based on two different pairs of rhombuses is called a Two Colour Penrose Tiling [10]. Regarding the systematic absences, a possible space group for main reflections could be $P \overline{\mathbf{1 0}} c 2$. Nevertheless, for the S1 satellite reflections, the $\boldsymbol{h}_{1} \boldsymbol{h}_{\mathbf{2}} \boldsymbol{h}_{\mathbf{2}} \boldsymbol{h}_{1} \boldsymbol{h}_{5}=\boldsymbol{h}_{\mathbf{3}}=\mathbf{2} n+\mathbf{1}$ rule is not verified (it holds for the S2 satellites). Interestingly however S1 reflections fulfil another systematic absences rule, namely $\boldsymbol{h}_{1} \boldsymbol{h}_{2} \overline{\boldsymbol{h}}_{\mathbf{2}} \overline{\boldsymbol{h}}_{\mathbf{1}} \boldsymbol{h}_{\mathbf{5}}: \boldsymbol{h}_{\overline{5}}=\mathbf{2 n + 1}$. This could beexplained by colour symmetry [11]. In this paper, however, the space group $P \overline{\mathbf{1 0}}$ is chosen (which explains 10/m Laue group choice). To keep the reflections-toparameters ratio reasonable, we decided to deflate the rhombuses (withrespect to those proposed by [9]) according to the inflation rules for Penrose tiling. The edge length of our structure units equals $12.1 \AA\left(2.43 \AA \cdot(3-\tau)^{0.5} \cdot \tau^{3}\right)$, the period $c$ along the quasi-tenfold axis equals $4.085 \AA$. The initial decoration of both pairs of rhombuses was the same. Figure 1 shows the initial positions of atoms and illustrates the refined TM concentration. Squares and circles distinguish atoms at $z=0.25 c$ and 
$z=0.75 c$ respectively. At some vertex positions both a circle and a square is visible. These are split positions - partial occupation in both layers is possible for these positions. Grey symbols denote TM atoms, light grey Al/TM atoms and white $\mathrm{Al}$ atoms. For simplicity atoms, whose occupation probability decreased to zero during the refinement process are not shown in the figure. The numbers indicated in Figure 1 are used in the section 'Optimization results' to list the occupation probabilities and TM concentration at each position. For the purposes of this paper the pair of rhombuses in Figure 1a will be called pair $A$ and in the Figure 1b pair B.
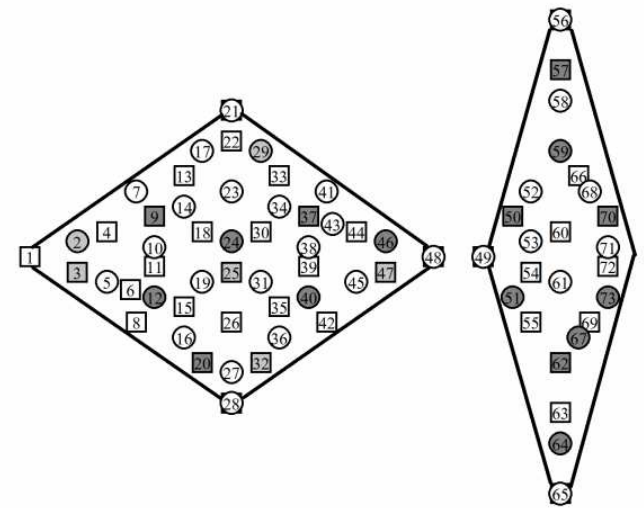

Figure 1a.- 'pair A' of structure units

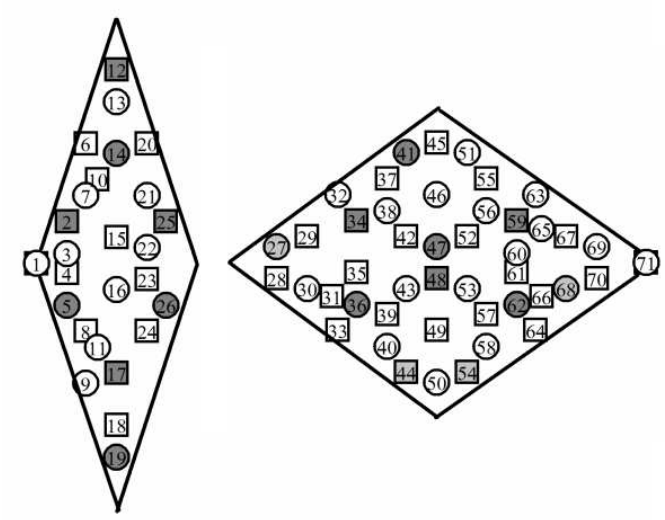

Figure 1b.-'pair B' of structure units.

\section{Structure factor}

The structure factor was calculated inphysical space on the basis of our statistical approach. The concept of average unit cell for Penrose tiling and a detailed derivation of the structure factor for any decoration is extensively discussed in [5]. Here we shall only show the resulting formula:

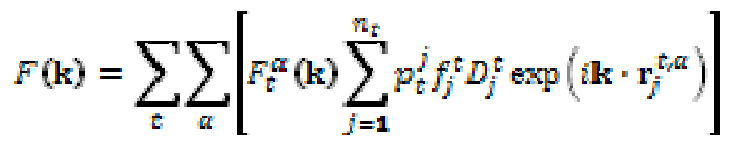

$F_{t}^{\alpha}(\mathbf{k})$ is the Fourier transform of a triangular probability distribution associated with a given structure unit at a given orientation. There are four structure units - two thick and two thin rhombuses (sum over $t$ ) and five possible orientations of each structure unit (sum over $\alpha$ ). The position of an atom $j$ in a given structureunit $t$ at orientation $\alpha$ is represented by $\mathbf{r}_{j}^{\alpha, t}, p_{j}^{t}$ is the weight of a given atom in a structure unit (calculated as a fraction of the atom, which is inside a given rhombus, e.g for group1 in the Figure 1a this weight is equal to ${ }^{72^{\circ}} \frac{{ }^{360}}{360}=\mathbf{0 . 2}$, etc.), $n_{t}$ is the number of atoms decorating a structure unit, $\mathbf{k}$ is a scattering vector and $f_{j}^{t}$ is the average atomic form factor. A formula for $f_{j}^{t}$ reads:

$f_{j}^{t}=p_{\text {calc }}^{j,}\left(p_{\mathbf{T M}}^{j, t} f_{T M}+\left(1-p_{\mathrm{TM}}^{j, t}\right) f_{A I}\right)$

$p_{\text {calc }}$ is the occupation probability of a given position by an atom, $p_{\mathrm{TM}}$ is the concentration of TM atoms at a given position, $f_{\mathrm{TM}}$ is the atomic form factor for TM 
atoms (weighted $\mathrm{Co}$ and $\mathrm{Ni}$ ), $f_{\mathrm{Al}}$ atomic form factor for $\mathrm{Al}$ atoms. $D_{l(s)}$ is the DebyeWaller factor:

$D_{j}^{t}=\exp \left(-\frac{1}{16 \pi^{2}}\left(k_{x}^{2}+k_{y}^{2}\right) b_{x y}-\frac{1}{16 \pi^{2}} k_{z}^{2} b_{z}\right)$

$b_{x y}$ is the average displacement parameter in the quasiperiodic plane, $b_{z}$ is the displacement parameter in the $z$-direction (periodic direction). A relation between these parameters and he square mean shift of the atom from the equilibrium position $\left\langle u_{x y(s)}^{z}\right\rangle$ reads:

$b_{x y(z)}=\mathbf{8} \pi^{2}\left(u_{x y(z)}^{2}\right)$

The space group chosen for the refinement is $P \overline{\mathbf{1 0}}$. This requires that all rhombuses within a given pair have the same decoration in all 5 possible orientations (i.e. all parameters describing atoms are the same in all 5 possible orientations). Therefore it is enough to describe a decoration of a given rhombus in just one chosen orientation.

\section{Optimized function}

We used a conjugated gradient minimization algorithm. The optimized function reads:

$$
\Delta=w R^{2}+P F_{1}+P F_{z}+P F_{3}+P F_{4}
$$

where:

$w R=\frac{\sqrt{\sum_{i}\left(\frac{F_{\mathrm{obs}}^{i}-F_{\mathrm{calc}}^{i}}{\sigma^{i}}\right)^{2}}}{\sqrt{\sum_{i}\left(\frac{F_{\mathrm{abs}}^{i}}{\sigma^{i}}\right)^{2}}}$

$$
\begin{aligned}
& P F_{1}=C_{1} \sum_{i=1}^{N_{\text {argans }}}\left(s_{\mathrm{TM}}^{i}+s_{\text {cale }}^{i}\right)
\end{aligned}
$$

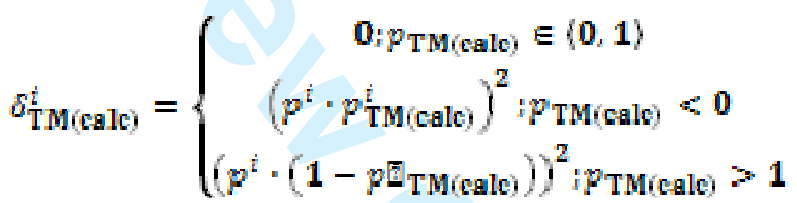

$P F_{\mathbf{z}}=C_{\mathbf{z}} \sum_{i=1}^{N_{\text {axzms }}} \delta_{\mathrm{sy}(\mathrm{z})}^{i}$

$P F_{3}=C_{\mathrm{s}}\left(d_{\mathrm{r}}-d_{\mathrm{t}}\right)^{2}$

$\sigma_{\mathrm{xy}(x)}^{i}=\left\{\begin{array}{c}0 ; b_{\mathrm{xy}(z)}>0 \\ \left(p^{i}-b \mathbb{Z}_{\mathrm{xy}(z)}\right)^{2} ; b_{\mathrm{xy}(z)}<0\end{array}\right.$

$$
P F_{\mathrm{A}}=c_{\mathrm{A}}\left(c_{\mathrm{r}}-c_{\mathrm{t}}\right)^{2}
$$

$F_{\text {obs }}$ and $F_{\text {calc }}$ are the amplitudes of observed and calculated structure factor respectively, $\sigma$ is the standard deviation of a structure factor amplitude, $N_{\text {atoms }}$ is the number of atoms decorating our structure units, $d_{\mathrm{r}}$ and $d_{\mathrm{t}}$ are the calculated and theoretical concentration of TM atoms respectively, finally $c_{\mathrm{r}}$ and $c_{\mathrm{t}}$ are the calculated and theoretical point density. $P F_{1}, P F_{2}, P F_{3}, P F_{4}$ keep the concentration of TM atoms, Debye-Waller coefficients, chemical composition and point density respectively in the physical boundaries. Numbers $C_{1}, C_{2}, C_{3}, C_{4}$, are the weights of a given component in the optimized function. 
Table 1. Refined occupation probabilities and TM concentrations.

\begin{tabular}{|c|c|c|c|c|c|c|c|c|c|c|c|c|c|c|c|c|c|}
\hline & \multicolumn{8}{|c|}{ Pair A } & \multicolumn{9}{|c|}{ Pair B } \\
\hline No & $\overline{p_{\text {calc }}}$ & $\overline{\mathrm{p}_{\mathrm{TM}}}$ & No & $\mathrm{p}_{\text {calc }}$ & $\mathrm{p}_{\mathrm{TM}}$ & No & $\mathrm{p}_{\text {calc }}$ & $\overline{p_{\mathrm{TM}}}$ & No & $\mathrm{p}_{\text {calc }}$ & $\mathrm{p}_{\mathrm{TM}}$ & No & $\overline{p_{\text {calc }}}$ & $\mathrm{p}_{\mathrm{TM}}$ & No & $\mathrm{p}_{\text {calc }}$ & $\overline{\mathrm{p}_{\mathrm{TM}}}$ \\
\hline 1 & 0.52 & 0.00 & 26 & 0.87 & 0.00 & 51 & 1.00 & 1.00 & -1 & 0.05 & 0.00 & 24 & 1.00 & 0.00 & 48 & 1.00 & 1.00 \\
\hline 2 & 0.55 & 0.58 & 27 & 1.00 & 0.00 & 52 & 1.00 & 0.00 & +1 & 0.11 & 0.00 & 25 & 1.00 & 0.86 & 49 & 1.00 & 0.00 \\
\hline 3 & 0.87 & 0.58 & -28 & 0.10 & 0.00 & 53 & 1.00 & 0.00 & 2 & 1.00 & 1.00 & 26 & 1.00 & 1.00 & 50 & 1.00 & 0.15 \\
\hline 4 & 1.00 & 0.00 & +28 & $0.2(2)$ & 0.00 & 54 & 1.00 & 0.00 & 3 & 1.00 & 0.00 & 27 & 0.81 & 0.47 & 51 & 1.00 & 0.24 \\
\hline 5 & 1.00 & 0.00 & 29 & 1.00 & 0.28 & 55 & 1.00 & 0.00 & 4 & 1.00 & 0.00 & 28 & 0.74 & 0.20 & 52 & 1.00 & 0.00 \\
\hline 6 & 0.29 & 0.00 & 30 & 1.00 & 0.00 & -56 & $0.2(5)$ & 0.00 & 5 & 1.00 & 1.00 & 29 & 1.00 & 0.00 & 53 & 1.00 & 0.00 \\
\hline 7 & 0.46 & 0.00 & 31 & 1.00 & 0.00 & +56 & $0.2(9)$ & 0.00 & 6 & 1.00 & 0.00 & 30 & 0.48 & 0.00 & 54 & 1.00 & 0.39 \\
\hline 8 & 1.00 & 0.00 & 32 & 1.00 & 0.69 & 57 & 1.00 & 1.00 & 7 & 1.00 & 0.00 & 31 & 0.50 & 0.00 & 55 & 0.52 & 0.00 \\
\hline 9 & 1.00 & 1.00 & 33 & 0.73 & 0.00 & 58 & 0.52 & 0.00 & 8 & 1.00 & 0.00 & 32 & 1.00 & 0.00 & 56 & 1.00 & 0.00 \\
\hline 10 & 1.00 & 0.00 & 34 & 1.00 & 0.00 & 59 & 1.00 & 1.00 & 9 & 1.00 & 0.00 & 33 & 1.00 & 0.00 & 57 & 1.00 & 0.00 \\
\hline 11 & 1.00 & 0.00 & 35 & 1.00 & 0.00 & 60 & 1.00 & 0.00 & 10 & 1.00 & 0.00 & 34 & 1.00 & 1.00 & 58 & 0.76 & 0.00 \\
\hline 12 & 1.00 & 1.00 & 36 & 0.90 & 0.00 & 61 & 1.00 & 0.00 & 11 & 0.53 & 0.00 & 35 & 0.76 & 0.00 & 59 & 1.00 & 1.00 \\
\hline 13 & 0.69 & 0.00 & 37 & 1.00 & 1.00 & 62 & 1.00 & 1.00 & 12 & 1.00 & 1.00 & 36 & 1.00 & 1.00 & 60 & 1.00 & 0.00 \\
\hline 14 & 1.00 & 0.00 & 38 & 1.00 & 0.00 & 63 & 0.50 & 0.00 & 13 & 0.77 & 0.00 & 37 & 1.00 & 0.00 & 61 & 0.63 & 0.00 \\
\hline 15 & 1.00 & 0.00 & 39 & 0.43 & 0.00 & 64 & 1.00 & 1.00 & 14 & 1.00 & 1.00 & 38 & 1.00 & 0.00 & 62 & 1.00 & 1.00 \\
\hline 16 & 0.46 & 0.00 & 40 & 1.00 & 1.00 & -65 & 0.25 & 0.00 & 15 & 1.00 & 0.00 & 39 & 1.00 & 0.21 & 63 & 1.00 & 0.00 \\
\hline 17 & 1.00 & 0.00 & 41 & 1.00 & 0.00 & +65 & 0.29 & 0.00 & 16 & 0.74 & 0.00 & 40 & 1.00 & 0.00 & 64 & 1.00 & 0.00 \\
\hline 18 & 0.28 & 0.00 & 42 & $0.6(3)$ & 0.00 & 66 & 1.00 & 0.00 & 17 & 1.00 & 1.00 & 41 & 0.77 & 0.68 & 65 & 0.65 & 0.00 \\
\hline 19 & 0.62 & 0.00 & 43 & 0.85 & 0.00 & 67 & 1.00 & 0.81 & 18 & 0.64 & 0.00 & 42 & 1.00 & 0.20 & 66 & 0.23 & 0.00 \\
\hline 20 & 1.00 & 0.89 & 44 & 1.00 & 0.00 & 68 & 1.00 & 0.00 & 19 & 1.00 & 1.00 & 43 & 1.00 & 0.00 & 67 & 1.00 & 0.28 \\
\hline-21 & 0.10 & 0.00 & 45 & 1.00 & 0.00 & 69 & 0.76 & 0.00 & 20 & 0.91 & 0.00 & 44 & 1.00 & 0.43 & 68 & 1.00 & 0.53 \\
\hline+21 & $0.2(2)$ & 0.00 & 46 & 1.00 & 1.00 & 70 & 1.00 & 1.00 & 21 & 1.00 & 0.00 & 45 & 1.00 & 0.00 & 69 & 1.00 & 0.27 \\
\hline 22 & 1.00 & 0.00 & 47 & 1.00 & 0.76 & 71 & 1.00 & 0.00 & 22 & 1.00 & 0.00 & 46 & 1.00 & 0.00 & 70 & 1.00 & 0.00 \\
\hline 23 & 0.64 & 0.00 & \pm 48 & 0.50 & 0.00 & 72 & 0.40 & 0.00 & 23 & 1.00 & 0.00 & 47 & 1.00 & 1.00 & -71 & 0.34 & 0.00 \\
\hline 24 & 1.00 & 0.91 & \pm 49 & 0.50 & 0.00 & 73 & 1.00 & 1.00 & & & & & & & +71 & 0.50 & 0.00 \\
\hline 25 & 1.00 & 0.74 & 50 & 1.00 & 1.00 & & & & & & & & & & & & \\
\hline
\end{tabular}

The Debye-Waller coefficient $b_{x y}$ i.e. the component in the quasiperiodic plane, is lower than $3 \AA^{2}$ for all atomic positions (for most of them it is lower than $2 \AA^{2}$ ), which is an acceptable value for inorganic materials and can be interpreted as arising solely from the thermal motion of atoms.However for some atomic positions the $b_{z}$ coefficient, i.e. the Debye-Waller factor component associated with $z$ direction, exceeds the value of $3 \AA^{2}$ (for some atoms even exceeds $5 \AA^{2}$ ). This could mean that the atomic layers of the structure are puckered rather than flat.Such an assumption is reasonable because of presence of diffuse maxima in the half-integer $h_{5}$ layers of the diffraction pattern [1]. In [12] even an $8 \AA$ superstructure for the basic Ni-rich is 
assumed. This means that the period along $z$ axis is doubled. However, there are no half-integer $h_{5}$ reflections in the diffraction pattern, thus in experiment only an average $4 \AA$ structure is observed. In this approach larger $b_{z}$ values result from overlapping layers of the superstructure having opposite $z$ displacements. In our paper only the average $4 \AA$ structure is investigated.

A Fourier map of the refined structure is shown in the Figure 2.Layers at $z=0.25 c$ and $z=0.75 c$ are superimposed.Grey lines show rhombuses chosen as structure units in the optimization process. Letters A and B correspond to pair A and pair B of our structure units respectively. Black lines show rhombuses chosen as structure units in [9], which are inflated (i.e. rescaled by $\tau$ ) with respect to the grey ones. It is clearly seen that the vertices of these rhombuses (black-lined) forming a Penrose tiling are decorated withquasitenfold clusters. TM atoms are likely to arrange around circles of about $12 \AA$ and $19 \AA$ in diameter, centred at the vertices of the Penrose tiling.

The shortest interatomic distance based on Patterson map analysis is about $2.43 \AA$. In the refined structure there are some distances shorter than this value. However distances shorter than $2.15 \AA$ exist only between pairs of atoms, for which the sum of occupation probabilities is lower than 1 . The distances in the range between $2.15 \AA$ and $2.43 \AA$ could be explained by the puckering of the atomic layers, which is not taken into account in the model and thus in the calculation of the interatomic distances.

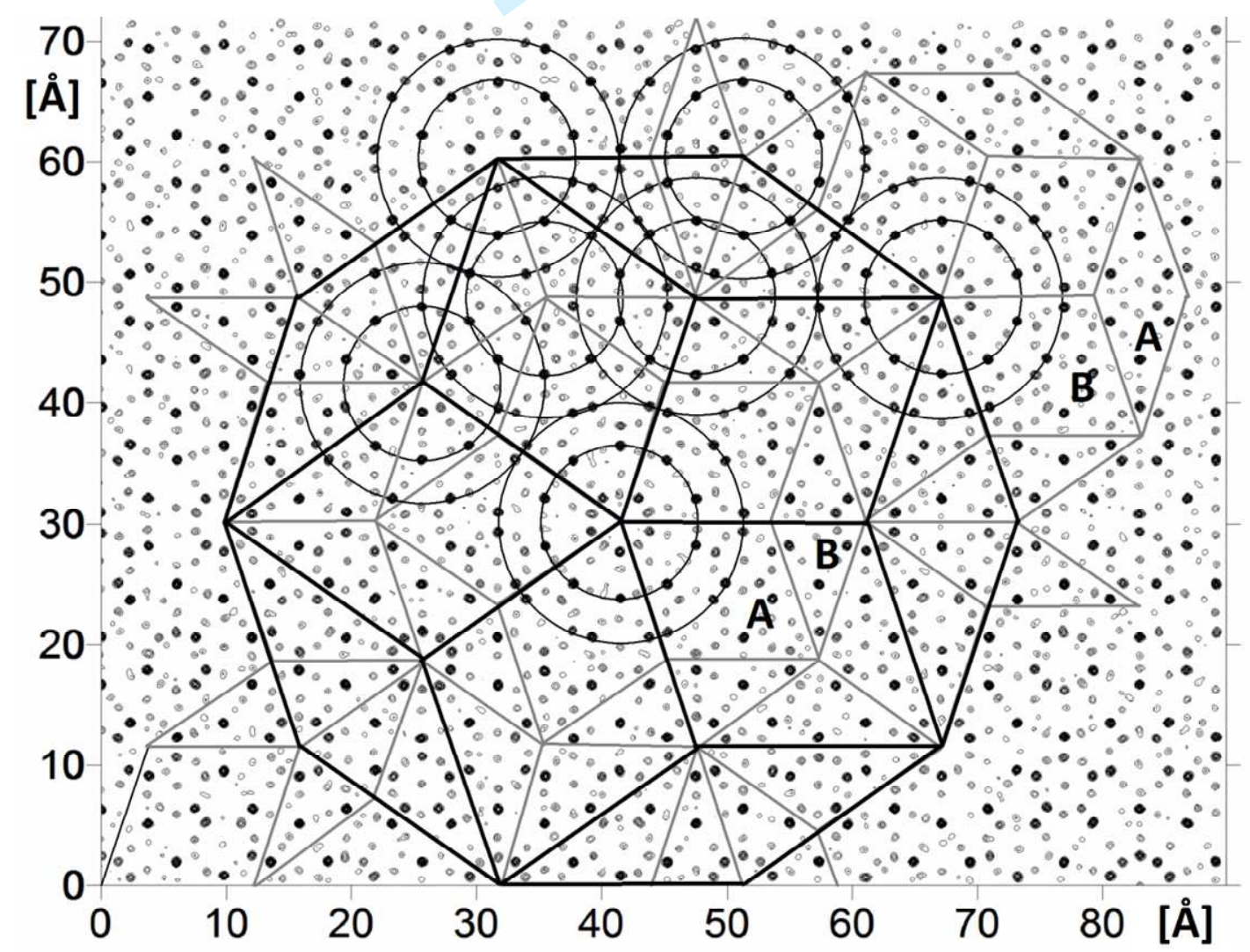

Figure 2. A Fourier map of the refined structure. Layers at $z=0.25 c$ and $z=0.75 c$ are superimposed. Letters A and B correspond to pair A and pair B of rhombuses from Figure 1. 
In the presented approach to structure refinement solely parameters related to physical space were refined, i.e. phasonic displacement parameters were not used neither individually for each atomic position nor globally. Extinction was also negligible. In the end we obtained $R=34.8 \%$ and $w R=6.0 \%$ for all 6843 reflections ( $R=29.5 \%, w R=5.9 \%$ for 6141 non-zero structure factor amplitudes), $R=26.8 \%$ and $w R=5.4 \%$ for all 4132 main + S1 reflections $(R=23.2 \%, w R=5.4 \%$ for 3790 non-zero structure factor amplitudes) and $R=13.3 \%, w R=3.6 \%$ for all 1387 main reflections $(R=12.1 \%, w R=3.6 \%$ for 1317 non-zero structure factor amplitudes). A detailed $R$ and $w R$ statistics is given in the Table 2.The values of $R$ and $w R$ for satellite reflections only are rather high. One should notice however, that $F / \sigma(\mathrm{F})$ ratio for these reflections is generally low e.g. there are 2711 S2 reflections but only for 386 of them $F$ is larger than $3 \sigma(F)$.

$F_{\text {calc }} / F_{\text {obs }}$ plot is shown in the Figure 4 . The chemical composition of the refined structure is $\mathrm{Al}_{70.4} \mathrm{TM}_{29.6}$, point density $0.0668 \AA^{-3}$ and overall density $4.04 \mathrm{~g} / \mathrm{cm}^{3}$. Chemical composition is almost equal to the measured one. Accurate density and point density values are known for the basic Ni-rich sample of the composition $\mathrm{Al}_{72} \mathrm{Ni}_{20} \mathrm{Co}_{8}$ [13] and are equal to $3.94 \mathrm{~g} / \mathrm{cm}^{3}, 0.0661 \AA^{-3}$ respectively.

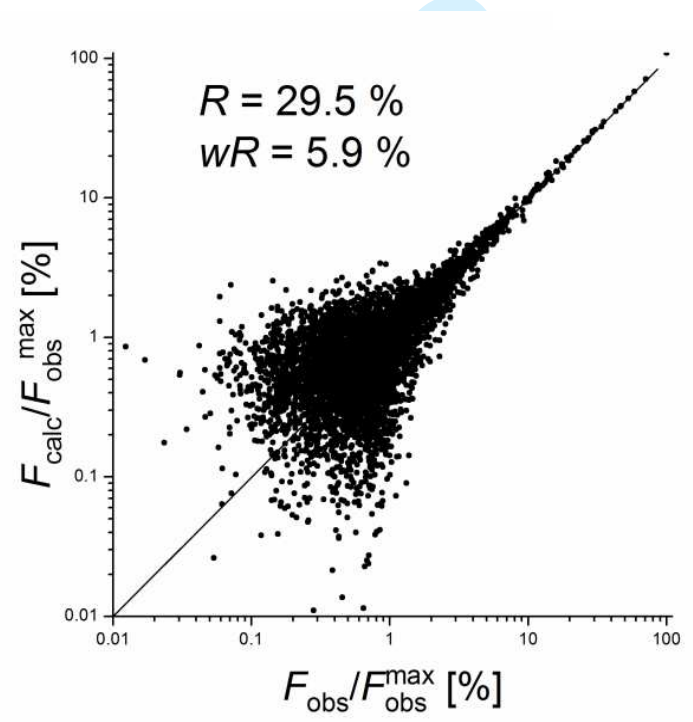

\begin{tabular}{|c|c|c|c|}
\hline \multicolumn{4}{|c|}{ Table 2. Structure factor statistics } \\
\hline No of refl. & & $R[\%]$ & $w R[\%]$ \\
\hline \multicolumn{4}{|l|}{ Main $+\mathrm{S} 1+\mathrm{S} 2$} \\
\hline All & 6843 & 34.8 & 6.0 \\
\hline$F>0$ & 6141 & 29.5 & 5.9 \\
\hline$F>\sigma_{\mathrm{F}}$ & 5091 & 25.3 & 5.8 \\
\hline$F>3 \sigma_{\mathrm{F}}$ & 2284 & 15.1 & 5.3 \\
\hline \multicolumn{4}{|l|}{ Main } \\
\hline All & 1387 & 13.3 & 3.6 \\
\hline$F>0$ & 1317 & 12.1 & 3.6 \\
\hline$F>\sigma_{\mathrm{F}}$ & 1198 & 10.8 & 3.6 \\
\hline$F>3 \sigma_{\mathrm{F}}$ & 870 & 8.1 & 3.5 \\
\hline \multicolumn{4}{|l|}{ S1 } \\
\hline All & 2745 & 49.1 & 19.2 \\
\hline$F>0$ & 2480 & 41.4 & 18.9 \\
\hline$F>\sigma_{\mathrm{F}}$ & 2091 & 36.4 & 18.6 \\
\hline$F>3 \sigma_{\mathrm{F}}$ & 1028 & 27.1 & 17.3 \\
\hline \multicolumn{4}{|l|}{$\mathrm{S} 2$} \\
\hline All & 2711 & 69.6 & 31.9 \\
\hline$F>0$ & 2344 & 56.8 & 30.1 \\
\hline$F>\sigma_{\mathrm{F}}$ & 1802 & 50.6 & 29.5 \\
\hline$F>3 \sigma_{\mathrm{F}}$ & 386 & 35.0 & 21.3 \\
\hline
\end{tabular}

Figure $4 . F_{\text {calc }} / F_{\text {obs }}$ plot.

\section{Conclusions}

In this paper we have described the first results of the structure analysis of decagonal Al-Ni-Co,superstructure type I (Edagawa phase). The refinement was conducted with only real space parameters, based on a decorated rhombic Penrose tiling model. Reasonable $R$ and $w R$ factors were obtained, density and point density are close do experimental values.In the resulting structure there is a certain degree of disorder within the structure units- thin and thick rhombuses (partial and/or mixed occupation). On the other hand, these rhombuses form the Penrose tiling, thus, in our approach, the structure is also perfectly long-range ordered. 


\section{Acknowledgements}

PawelKuczera's PhD fellowshipat the Laboratory of Crystallography, ETH Zurich is financed by SciexNMS ${ }^{\text {ch }}$.

This work has been supported by Ministry of Science and Higher Education of Poland.

Structure optimization calculations was performed in Academic Computer Center CYFRONET AGHon SGI Altix 3700 computerand financed under computational grant MNiSW/SGI3700/AGH/019/2010.

We thank the staff of the Swiss-Norwegian beam line (SNBL) at the European Synchrotron Radiation Facility (ESRF) in Grenoble, France, for their support.

\section{References}

[1] S. Katrych, W. Steurer, Z. Kristallogr. 219 (2004) 606-613..

[2] K. Edagawa, M. Ichihara, K. Suzuki., S. Takeuchi, Phil. Mag. Lett.66 (1992) $19-25$.

[3] T. Haibach, A. Cervellino, M.A. Estermann, W. Steurer, Phil. Mag. A79 (1999) 933-942.

[4] B. Kozakowski, J. Wolny,Phil. Mag.86 (2006) 549-555.

[5] B. Kozakowski, J. Wolny, Acta Cryst. A66 (2010) 489-498.

[6]J. Wolny, B. Kozakowski, P. Kuczera, H. Takakura, Z. Kristallogr.223 (2008) 847-850.

[7] P. Kuczera, B. Kozakowski, J. Wolny, W. Steurer, J. Phys.: Conf. Ser. 226 (2010) 012001.

[8] V. Petricek et al., ActaCryst. A 45 (1989), 63-75.

[9] A. Yamamoto, S. Weber, Phys. Rev. Lett.78 (1997) 4430-4433.

[10] A. Yamamoto, H. Takakura, E. Abe, Phys. Rev. B 72 (2005) 144202.

[11] X. Z. Li, J. M. Dubois, K. H. Kuo, Phil. Mag. Lett. (1994) 69 93-98.

[12] A. Cervellino A, T. Haibach, W. Steurer, Acta Cryst. B 58 (2002) 8-33.

[13] P. J. Steinhardt, H. C. Jeong, K. Saitoh, M. Tanaka, E. Abe, A.P. Tsai, Nature396 (1998) 55-57. 